UDC 004.942:532.1

\title{
On Cartesian grids in some adaptive algorithms of aerodynamics*
}

\section{A.L. Afendikov, V.S. Nikitin**}

Keldysh Institute of Applied Mathematics, RAS, Russian Federation

The paper describes several approaches to adaptation of Cartesian grids in fluid and gasdynamics problems. It is demonstrated that the filter based on non-uniform B-splines is more appropriate for this class of problems.

Keywords: gasdynamics; spatial grids; discretization; algorithm; parallel implementation.

Introduction. Hydro and gas dynamics have critical scientific and technical applications. Moreover, along with experimental and rigorous mathematical results, mathematical modeling, based on the use of parallel supercomputer systems, plays an increasing role. Often, the problems under investigation are characterized by a strong difference in space-time scales, for the solution of which not only high-precision numerical methods are required, but also very detailed spatial grids in geometrically complex domains whose boundaries can depend on time. For such tasks, up to half the time necessary to simulate and perform the calculation is spent on generating the initial grid and then modifying it (usually by hand) in order to obtain a solution of adequate quality. Therefore, one of the urgent problems is the development of methods for automatic adaptation of grids.

There is no strict mathematical theory for mathematical models describing these phenomena and processes that distinguishes suitable functional compacts in which solutions of the corresponding initial-boundary value problems lie. Therefore, the considerations underlying the approximation of solutions and the construction of discrete models and algorithms should serve to achieve a compromise between physical representations about the nature of the solutions (first of all, smoothness and multi-scale), the optimality in the number of operations, the possibility of massive parallelization, and also the specific architecture and element base (CPU, GPU) of the supercomputer used for calculations.

In the numerical solution of hydrodynamic problems, discretization is used when the domain of the solution is divided into countable cells by means of an appropriately selected grid, and the continuous problem reduces to a discrete approximation approximating the differential operators and boundary conditions. Currently, there are several approaches to this task.

The first is the standard method of connected grids, the second is the method of chimera grids, where the main grid is built throughout the calculation area, and the second-level grid is constructed separately around each solid object over the main grid. The third approach is the method of Cartesian grids, where the design area is chosen in the simplest form, for example, in the form of a cube, in which a regular Cartesian grid is constructed [2]-[4]. Surfaces of bodies in the current region cross

\footnotetext{
* The research is done on the grant of the RSF 17-71-30014.

** E-mail: andre@spp.keldysh.ru, andre@keldysh.ru.
} 
individual cells. Thus, there are two types of counting cells: normal cells, entirely in the region occupied by the gas, and truncated, having the shape of a certain polyhedron, depending on the topology of the intersection. The discretization of the equations is performed for cells of both normal and truncated type by a finite volume method ([1]).

Despite the presence of well-known commercial (StarCD, StarCCM +, CFX, FLuent) and open (Open FOAM) CFD packages on unstructured grids, algorithms on Cartesian grids based on the immersed boundaries method retain a number of competitive advantages. This is primarily due to the simplicity of the grid generation and the discretization of the equations of motion, as well as the opening possibilities of using parallel supercomputers.

The following format for describing a two-dimensional grid with the possibility of two-level local adaptation was used in [5]. Each source cell can be divided into four equal sub-cells by divisions in half in each direction. The analysis was conducted globally across the entire grid.

The locality of the wavelet transform was used in [6]. The rectangular grid of size $M \times N$ with cells allowing a multilevel partition was taken. Each cell is described by its level (with zero for a basic grid of size $M \times N$ ). A virtual position at this level is a pair of parameters indicating which row and column the cell would occupy in the case of full filling of this level. In addition, it has a flag field indicating whether a given cell is final (in the corresponding end leaf of the tree) and the solver is applied or subdivided into 4 cells of the next level. For a subdivided (parent) cell, there are pointers to the descendants of the given cell (i.e., the 4 cells to which this one is sub-divided). For the final cell, these pointers are empty, but there is a pointer to the structure that describes the real physical cell. It stores the cell coordinates and gas-dynamic characteristics inside it. In the case of a fictitious cell (broken), this pointer is, of course, zero. This data structure allows us to describe adaptive grids of completely different configurations without using unnecessary memory.

For automatic local grinding and coarsening of the grid in accordance with local flow properties, a grid analyzer based on wavelet analysis with wavelets constructed from uneven Bsplines is used.

1. Algorithm for traversing the grid and searching for neighbors. By passing the square array of trees described above is accomplished by a simple procedure: we bypass the double cycle for all elements of the basic grid. In the case where the current cell is finite, we apply the objective function to it, otherwise we call it for all four descendants of this cell.

To search for neighbors it is convenient to use one more feature of the meshes of the described format: a cell of the 1 st level with indices $(i, j)$ necessarily has its parent - a cell with indices $([i / 2],[j / 2])$

from zero, $i=0, \ldots, M-1 ; j=0, \ldots, N-1$; Then, for example, for the case of two levels neighbors of the current cell for each axe can be obtained by a simple search based on half division and multiplication of the indices.

Gas-dynamic calculation scheme on an unstructured grid. If the calculated stack consists of cells of different levels simultaneously, it becomes non-conformal and the total flow through the cell is computed by a more complex formula: 


$$
\underset{|v|, i, j}{Q \quad n+1}=\underset{|v|, i, j}{Q}{ }^{n}-\frac{\Delta t}{V_{|v|, i, j}}\left[\sum_{\sigma x} s_{\sigma} \sigma_{\sigma}+\sum_{\sigma y} s_{\sigma} \sigma_{\sigma}\right]
$$

$Q^{n}$

where $|v|, i, j$ is the value of the vector of conservative variables in the cell of level $|v|$ with virtual indices $(i, j)$ at this level in the time step with the number $n$. Flows multiplied by the corresponding areas of the parts of the boundary, along each direction, are summed along the entire cell boundary. $(\rho, U, U$ When the grid is milled, all descendants carry the value of the primitive vector of variables $\left(\rho, U_{x}, U_{y}, p\right)$, when four cells are combined into one, the conservative vector of variables in the cell takes the arithmetic mean of the conservative vectors in the descendant cells.

\section{Indicators of the smoothness of the solution given on an uneven grid on the basis of} wavelet analysis. When working with multilevel computational grids, there is a need for indicators that signal the need to rebuild the mesh. In [5-7] for the analysis the re-interpolation is used on a uniform grid. In this paper the approach where the analysis is carried out directly on an nonuniform grids, with the use of the de Boor formula for constructing B-splines on an non uniform grid [8].

Let us compare results of the two-dimensional modeling of Sedov's problem on a strong explosion with a backpressure.

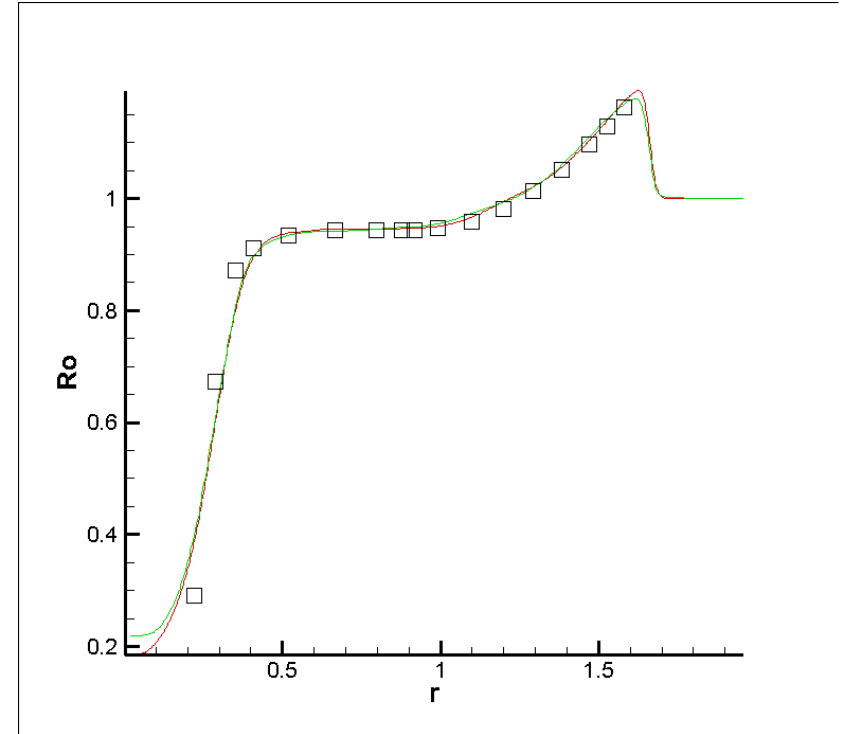

Fig. 1. The dependence of the density on the radius in the two-dimensional problem of a strong explosion with backpressure. The squares correspond to the data from [9]. The red indicates the dependence for the calculation with a uniform, and the green with non-uniform analyzers.

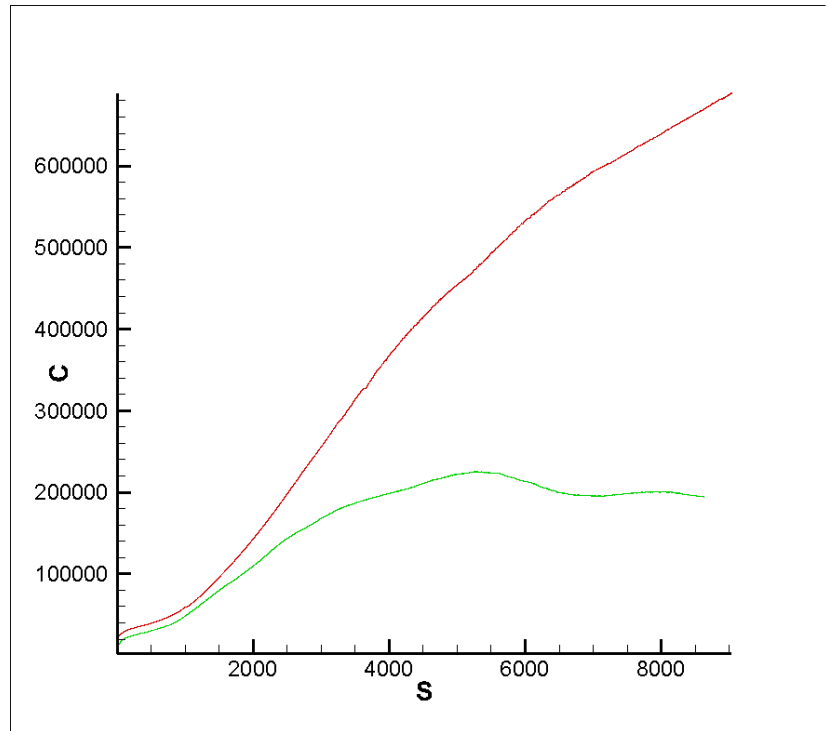

Fig. 2. The dependence of the number of cells on the step number. 
Conclusion. The given data demonstrate the practical coincidence of the results of calculations with a gain in the calculation time, which can be noticeably an order of magnitude, due to a more efficient non-uniform analysis and a multiple reduction in the number of computed cells. This is due to the fact that a uniform analyzer does not show adequate results in areas with local mesh unevenness. As a result of overestimation of the smoothness indicator of the solution, many extra cells are formed, and in addition, in areas where the detailed grid is no longer required, its coarsening is inefficient, which is demonstrated by a constant increase in the number of cells in the uniform case.

\section{References}

1. Afendikov, A.L. Adaptive wavelet algorithms for solving problems of hydro- and gas dynamics on Cartesian grids. Moscow: KIAM RAS, 2016. 228 p.

2. Chetverushkin, B.N., Churbanova, N.G., Trapeznikova, M.A., Sukhinov, A.A., Malinovskij, A.A. Adaptive cartesian mesh refinement for simulating multiphase flows in porous media. Computational methods in applied mathematics. Vol.8 (2008). No.2, pp.101-115

3. Khokhlov, A.M. Fully threaded tree for adaptive refinement fluid dynamics simulations. arXiv: astro-ph / 9701194v1 25 Jan 1997.

4. Hartmann, D., Meinke, M., Schroder, W. A strictly conservative Cartesian cut-cell method for compressible viscous flows on adaptive grids. Computer Methods in Applied Mechanics and Engineering, 2011. pp. 1038-1052.

5. Afendikov, A.L., Merkulov, K.D., Plenkin, A.V. Dynamic local adaptation of grids based on wavelet analysis in problems of gas dynamics // Preprints of the KIAM RAS. 2014. No. 99. 26 p. http://library.keldysh.ru/preprint.asp?id=2014-99

6. Afendikov, A.L., Merkulov, K.D., Plenkin, A.V. A Comparative Analysis of Approaches to Numerical Simulation of Gasdynamic Flows on Two-Level Adaptive Computational Grids // Preprints of the KIAM RAS, 2015. № 95. 24 p. URL: http://library.keldysh.ru/preprint.asp?id=201595

7. Afendikov, A.L., Lutsky, A.E., Menshov, I.S., Merkulov, K.D., Plenkin, A.V., Khankhasaeva, Ya.V. Algorithm of dynamic local adaptation of grids based on wavelet analysis using free boundary method // Preprints of the KIAM RAS. 2015. No. 94. 20 p. http://library.keldysh.ru/preprint.asp?id=2015-94

8. C. de Boor. A Practical Guide to Splines (revised ed.), Springer-Verlag, New York. 2001.

9. Kestenboim, Kh.S., Roslyakov, G.S., Chudov, L.A. Point explosion: calculation methods: tables / AS of the USSR, Institute of Problems of Mechanics. M.: Science, 1974, 255 p.

\section{Authors:}

Afendikov Andrei Leonidovich, Deputy Director of Research of Keldysh Institute of Applied Mathematics Russian Academy of Sciences (4 Miusskaya pl., Moscow, Russian Federation), Doctor of Science in Physics and Maths.

Nikitin Vyacheslav Sergeyevich, Keldysh Institute of Applied Mathematics Russian Academy of Sciences (4 Miusskaya pl., Moscow, Russian Federation) 
УДК 004.942:532.1

\section{О декартовых сетках в некоторых адаптивных алгоритмах аэродинамики*}

\section{А.Л. Афендиков, В.С. Никитин ${ }^{* *}$}

Институт прикладной математики им. М.В. Келдыша РАН, Москва, Россия

При численном решении задач гидрогазодинамики используется адаптивная дискретизация, когда область решения разбивается на счетные ячейки с помощью надлежащим образом подобранной сетки, а непрерывная задача сводится к дискретной, аппроксимирующей дифференциальные операторы и краевые условия.

Ключевые слова: гидрогазодинамика; пространственные сетки; дискретизация; алгоритм; параллельная реализация.

\section{Авторы:}

Афендиков Андрей Леонидович, заместитель директора по научной работе Института прикладной математики им. М.В. Келдыша Российской академии наук (125047, Москва, Миусская пл., д. 4), доктор физико-математических наук.

Никитин Вячеслав Сергеевич, Институт прикладной математики им. М.В. Келдыша Российской академии наук (125047, Москва, Миусская пл., д. 4)

\footnotetext{
* Работа поддержана грантом РНФ 17-71-30014.

**E-mail: andre@spp.keldysh.ru, andre@keldysh.ru.
} 